2 SLE activity as measured by the Polysymptomatic Distress (PSD) Scale.

Methods Lupus patients meeting ACR or SLICC criteria in a university lupus registry completed the PSD. SLEDAI scores were also recorded. Patients with $\geq 2$ clinical visits over a 52week period were included. Groups were selected based on mean, indicating severity of symptoms, and the standard deviation, indicating variability of symptoms, of PSD scores across visits. Differences across groups were assessed with chi-square and ANOVA tests.

Results The study included 204 patients. Four Type 2 SLE activity groups were identified (figure 1): chronic low $(n=71$; $35 \%)$, variable low $(n=31,15 \%)$, chronic high $(n=31,15 \%)$, and variable high $(\mathrm{n}=71,35 \%)$.

Patients in the chronic low and variable low Type 2 groups had similar demographics, with two-thirds being Black and 54 and $68 \%$, respectively, having a history of nephritis. The chronic low Type 2 group had stable minimal Type 2 SLE, with an average PSD score of 3.7 that ranged from 2.8 to 4.7. Similarly, these patients had minimal Type 1 SLE activity, with average clinical SLEDAI scores of 0.7 . The variable low Type 2 group had higher PSD scores (average: 4.5), ranging from 1.8 to 7.7 , as well as higher SLEDAI scores than the chronic low Type 2 group, with $\sim 25 \%$ having a SLEDAI $\geq 8$.

Patients in the chronic high and variable high Type 2 groups also had similar demographics with half being Black and two-thirds having no history of nephritis. Additionally, both groups had average SLEDAI scores of 4. Patients in the chronic high Type 2 group had stable high PSD scores ranging on average from 10.3 to 12.7 . Patients in the variable high Type 2 group had average scores of 14.1 with a larger range: 10 to 18.5 .

Conclusion These findings indicate that patients with lupus differ in their Type 2 SLE activity. One-third of patients had constant high Type 2 activity, and about half had fluctuating Type 2 symptoms. Future studies will determine if this fluctuation is due to inflammation or non-inflammatory etiologies such as perceived stress, extent of social support, PTSD, illness perception, or resilience factors.

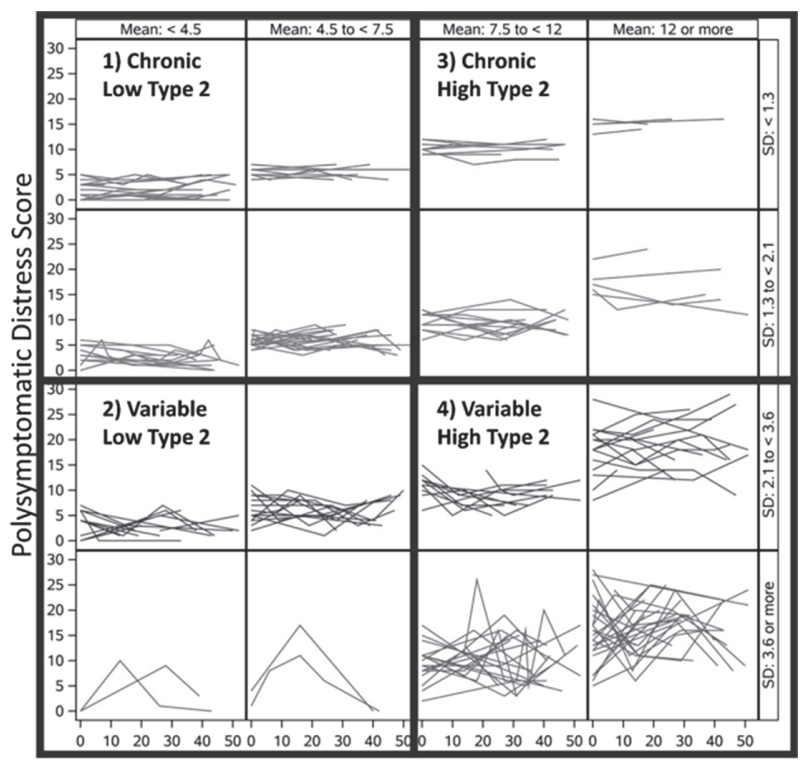

Weeks Follow-Up

Abstract 1001 Figure 1 Change in Polysymptomatic Distress Scores over time in four Type 2 SLE trajectory groups

\section{2 \\ THE MITIGATING EFFECTS OF SELF-EFFICACY ON PAIN INTERFERENCE DIFFER BY DEPRESSION IN BLACK WOMEN WITH SYSTEMIC LUPUS ERYTHEMATOSUS}

Cristina Drenkard. Division of Rheumatology, Department of Medicine, Emory School of Medicine, Atlanta, Georgia, USA

\subsection{6/lupus-2021-lupus21century.42}

Background Pain is a pervasive and distressful symptom in patients with SLE, particularly among high-risk individuals. Self-efficacy can mitigate the negative impact of pain on daily activities. However, depressed patients face challenges to control pain. We examined whether self-efficacy affects pain interference in Black women with SLE and whether depression alter those relationships.

Methods Baseline PROMIS measures of Pain interference, Selfefficacy to manage symptoms (SEMS), Self-efficacy to manage medications and treatments (SEMMT), and Depression were transversally collected among Black women with SLE who participated in the Women Empowered to Live with Lupus (WELL) Study. We examined the relationship between Pain interference and self-efficacy (SEMS and SEMMT), and the interaction of depression with self-efficacy, after adjusting for confounders.

Results Of 699 participants, 143 (21\%) were 18-34, 329 (47\%) 35-54, and $227(33 \%) \geq 55$ years old; 262 (38\%) attained shigh school, 226 (32\%) some college, and 211 (30\%) $\geq$ bachelor's degree. Pain interference declined by 2.8 points per 5-point increase in SEMS (slope $=-0.556$, pvalue $<0.001)$ and by 1.4 points per 5 -point increase in SEMMT (slope $=-0.282$, p-value $<0.001$ ). The table depicts the adjusted slopes and adjusted means of Pain interference on SEMS and SEMMT by depression severity. Depression showed a statistically significant interaction with SEMS $\left(\mathrm{R}^{2}=0.41\right.$, $\mathrm{p}$ value for the interaction $=0.05)$. Pain interference was inversely and significantly associated with SEMS in patients without depression (adjusted slope $=-0.196$, p-value $<0.001$ ); however, the association was not statistically significant in those with mild depression (adjusted slope $=0.035$, p-value $=0.7$ ), nor those with moderate/severe depression (adjusted slope $=-0.080, \mathrm{p}$-value $=0.3)$. No significant mean difference ( $\mathrm{p}$-value $=0.11)$ was observed among patients without depression (adjusted mean=56.9), mild depression (adjusted mean $=58.2$ ), and moderate/severe depression (adjusted mean=58.9). Although depression did not show an overall statistically significant interaction effect with SEMMT $\left(\mathrm{R}^{2}=0.40\right.$, $\mathrm{p}$-value for interaction $\left.=0.06\right)$, we did observe a statistically significant mean difference ( $\mathrm{p}$-value $=0.003$ ) among patients without depression (adjusted mean=56.2), mild depression (adjusted mean $=58.0$ ), and moderate/severe depression (adjusted mean $=59.2$ ).

Conclusion Self-efficacy (to manage symptoms and to manage medications and treatments) was inversely related to pain interference in Black women with SLE. However, depression altered those relationships, particularly decreasing the potential benefits of self-efficacy to manage symptoms of pain interference. Depression is a highly prevalent and often underdiagnosed comorbidity in patients with SLE; consequently, untreated depression may limit the potential benefits of selfmanagement interventions that rely on self-efficacy to achieve better pain control in this population. Programs designed to build self-efficacy should consider these findings to determine how to maximize intervention effectiveness.

Trial Registration Number NCT02988661 